\title{
Transmission Performance Research of Two Kinds of Visible Light Duobinary Signals
}

\author{
Yue Zhou ${ }^{1}$, Yufeng Shao ${ }^{* 1, a}{ }^{\text {,Zhifeng Wang }}{ }^{1}$, Junyi Zhou ${ }^{1}$, Changxiang Li $^{1}$, Wenzhe Ma ${ }^{1}$, \\ XingPing $\mathrm{Ji}^{1}$ and Jianjun Wang ${ }^{1}$ \\ ${ }^{1}$ Faculty of Engineering, Shanghai Polytechnic University, Shanghai, China \\ ayfshao@sspu.edu.cn
}

Keywords: VLC, DA-ODB, LDF-ODB, Receiver sensitivity.

\begin{abstract}
Visible light communication (VLC) as a kind of short-range wireless optical communication technology, plays dual role of green illumination and data communication, and gets more attention. At the same time, since optical duobinary (ODB) modulation technique has good dispersion resistance, high spectral efficiency and simple realization, it can be used in the VLC systems. Two different optical duobinary modulation schemes (DA-ODB and LDF-ODB) are used to realize $10 \mathrm{~m}$ wireless transmission of visible light with $550 \mathrm{~nm}$ wavelength. The results show that when the BER (bit error rate) of received signals is $10^{-9}$, the receiver sensitivity in the LPF-ODB system is $-35.9 \mathrm{dBm}$, and the receiver sensitivity in the DA-ODB system is $-38.3 \mathrm{dBm}$ which has better reception performance. The above results verify visible light duobinary signals have potential application value in the future wireless optical access systems.
\end{abstract}

\section{Introduction}

Light emitting diode (LED) compared to the incandescent and fluorescent lamps, which has long life, high light efficiency, low power consumption, no radiation, good security, high reliability, is known as the "green lighting" and develop rapidly[1]. White LED VLC is the fusion of optical communication and wireless communication based on two advantages, and conform to the white LED devices as the development trend of the next generation of solid green lighting source, and it is a high-speed, flexible new green communication technology[2]. The wavelength range of VLC is from $380 \mathrm{~nm}$ to $780 \mathrm{~nm}$ LED visible light optical source. Due to the high frequency sensitivity, and good linear modulation characteristics, therefore LED can be used to realize data transmission[3-4]. Moreover, the communication circuit can be integrated with the driving circuit of the LED lamp, and is very promising in the fields of lighting and communication, visual signal and data transmission, display and data communication, indoor positioning, etc.. Japan's visible light Communications Alliance conducted a preliminary study of visible light communication firstly, Asia, Europe, and the wireless world research forum is also working in VLC research[5-6].Optical duobinary modulation and demodulation is widely used in optical communication system, because of the advantages such as the good anti-dispersion performance, high spectral efficiency and simple implementation 
technology[7]. According to the reported literature, optical duobinary modulation can be used in the visible light communication system application. Considering the optical duobinary signal has a kind of partial response signal features (according to Nyquist second standards)[8], which has good performance in coding theory (can reduce inter-symbol interference effects, and can enhance the ability of error detection and recovery)[9], so we intend to carry out applied research in visible light communication system.

\section{System Principle}

In this paper, the model of VLC system is based on Optiwave software and MATLAB, using two optical duobinary signals of different structure for transmission, and comparing their transmission performances. The system block diagram is shown in Fig. 1.The rate of the simulation experiment system is set to be $10 \mathrm{Mb} / \mathrm{s}$, and using intensity modulation/direct detection(IM/DD) method.

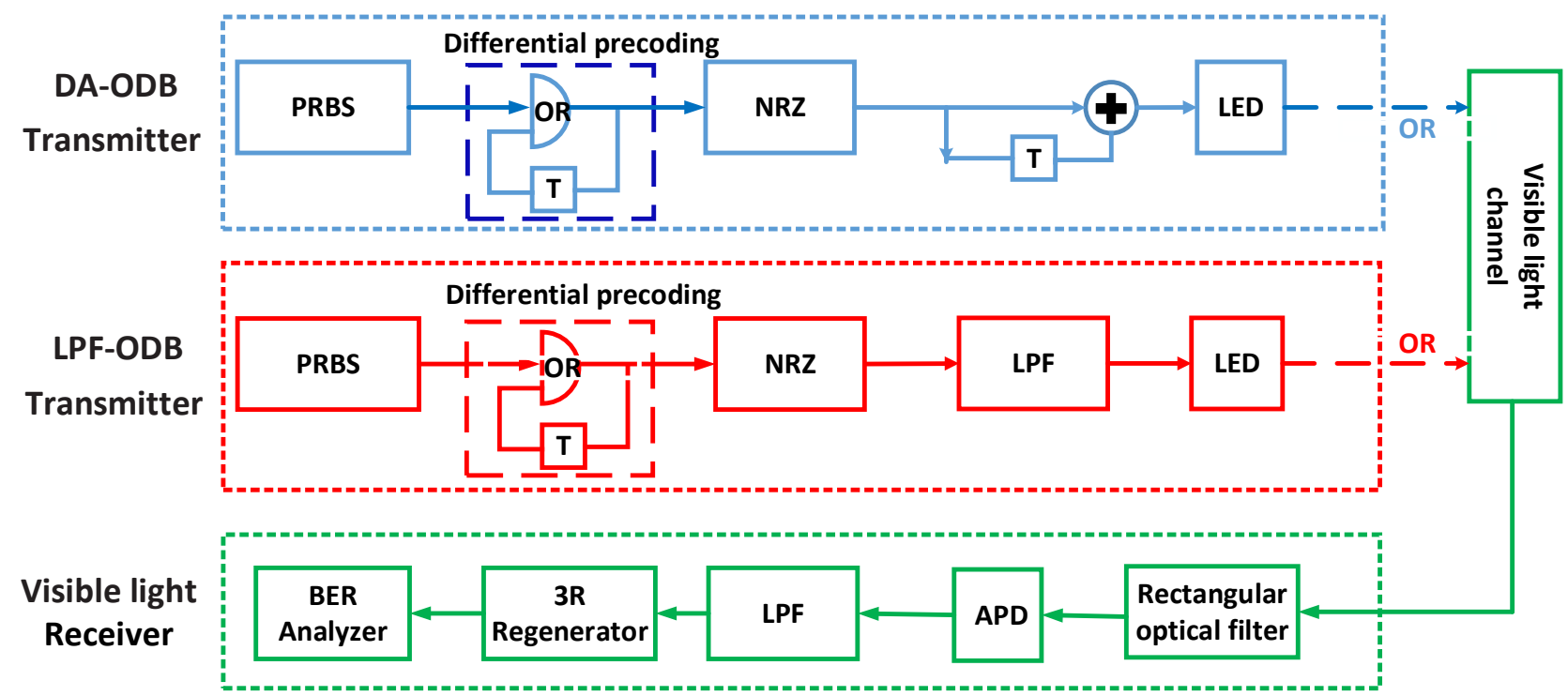

Fig.1 Block diagram of VLC system

In the ODB transmitter, the generated pseudo-random sequence should be pre-differential modulated firstly, because the optical duobinary modulation has a modulation characteristic of partial response. It is necessary to pre-encode firstly, which can prevent error propagation. The experiment uses XOR gates and delay[9], implement the differential pre-encoding. In the first transmitter, using the LPF-ODB modulation, to realize the implement of electric double binary coding. By using a low pass filter instead of the delay adding unit. In the second transmitter, a conventional DA-ODB modulation is used to implement the electrical double binary encoding through a delay and adder. The final modulated signal directly drives the LED module to form an optical signal that varies with the intensity of the optical carrier and is transmitted in the visible light channel. The wavelength of the LED is $550 \mathrm{~nm}$ (green light LED), and the transmission power is controlled to be $500 \mathrm{~mW}$. In the visible light transmission channel, the emission and reception aperture areas are set to be $0.8 \mathrm{~cm}$ and $1 \mathrm{~cm}$ respectively, and the attenuation parameter is set to be $5 \mathrm{~dB} / \mathrm{km}$. In the receiver mainly includes the optical filter, photodetector, low pass filter and 3R re-generator, as shown in Fig. 2, visible light receiver. The rectangular optical filter is adapted to filter the excess visible light noise. The photodetector is consider to use avalanche photodiode (APD), the response is $0.9 \mathrm{~A} / \mathrm{W}$, the dark 
current is $10 \mathrm{nA}$. The cut-off frequency of low-pass filter is 0.75 times the bit rate. Finally, the $3 \mathrm{R}$ re-generator is used for the recovery of original signal. Measuring devices include the oscilloscope, the RF spectrum analyzer, the optical power meter, the eye diagram analyzer and the BER analyzer.

\section{Results Analysis}

In VLC system, the electrical spectrum, transmission waveform and eye diagrams of the two ODB modulation schemes after transmission is shown in Fig. 2 and Fig.3. Compared to the electrical spectrum of Fig. 2 (a) and Fig. 3 (a), it is found that the DA-ODB scheme has a significant decrease in the frequency of $10 \mathrm{MHz}$, while the power attenuation of the LPF-ODB scheme is relatively flat, indicating that the side-lobe power suppression capability of the LPF-ODB scheme is worse than the DA-ODB scheme. Comparing the transmission waveform in Fig. 2 (c) and Fig. 3 (c), it it found that while using the low pass filter method in the LPF-ODB modulation, because of the non-ideal characteristic of low pass filter, which the cut off frequency has a certain width, and ultimately improve the BER and reduce the transmission performance of the system. Compared with the two waveforms before and after transmission, the waveform quality of the DA-ODB scheme is obviously better than that of the LPF-ODB scheme, and the waveform becomes uneven due to the introduction of noise and attenuation in the visible channel.The eye diagrams of two different optical binary modulation schemes are shown in Fig. 2 (b) and Fig. 3 (b). The eye opening degree of the DA-ODB scheme is significantly larger than that of the LPF-ODB scheme, which reflects the stronger ability the DA-ODB scheme.

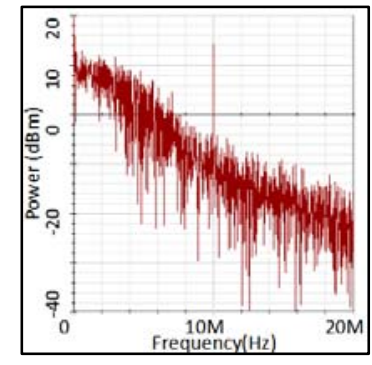

(a) Electrical spectrum transmission)

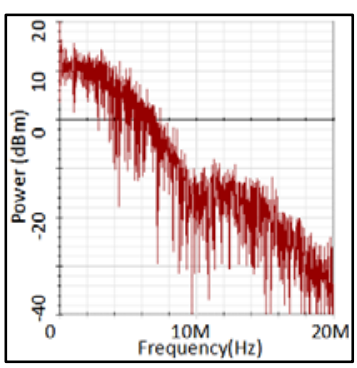

(a) Electrical spectrum transmission)
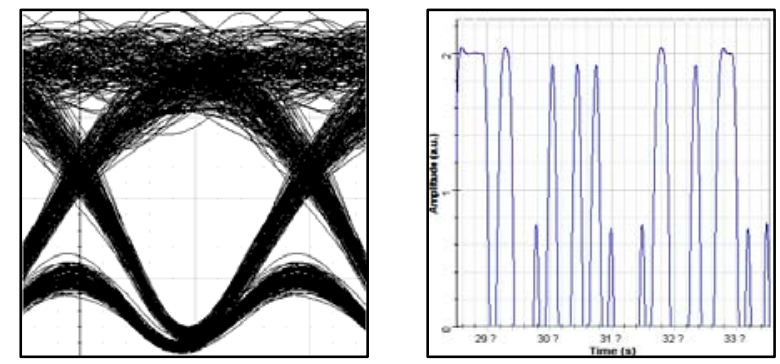

(b) Eye diagram (c) Time waveform (BTB)

Fig. 2 Result diagrams of LPF-ODB scheme
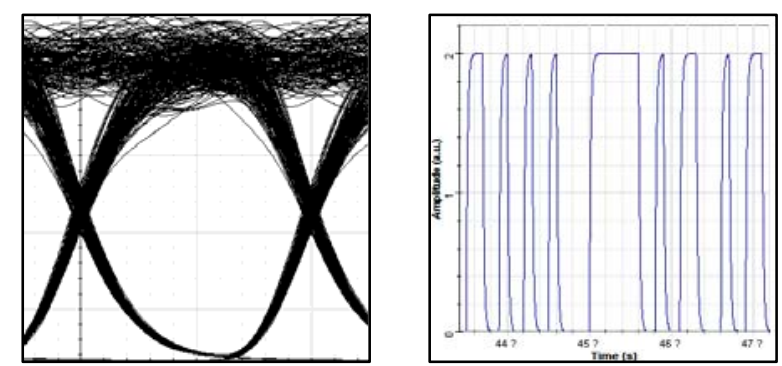

(b) Eye diagram (c) Time waveform (BTB)

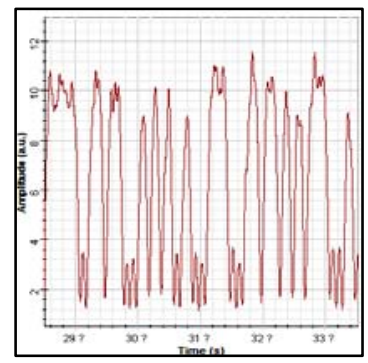

(d) Time waveform (after

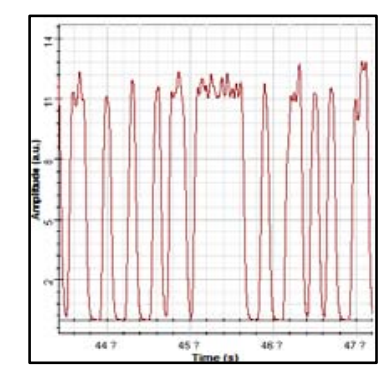

(d) Time waveform (after

\section{$\begin{aligned} & \text { Fig. } 3 \text { Result diagrams of DA-ODB scheme } \\ & \text { The received power and BER are shown in Fig. } 4 \text {, according to the Q valu }\end{aligned}$}

The received power and BER are shown in Fig. 4, according to the Q value of 6, BER is $10^{-9}$ as the system maximum tolerance boundary condition [10]. By comparing the received power of the two systems, it is found that the received power of the LPF-ODB scheme is $-35.9 \mathrm{dBm}$, as shown in Fig. 4 (a) (b), and the received power of the DA-ODB scheme is $-38.3 \mathrm{dBm}$, which is $2.4 \mathrm{dBm}$ lower than the 
former. Comparing with the received power of the two modulation schemes, it is found that the visible light system with DA-ODB scheme has higher receiving sensitivity.

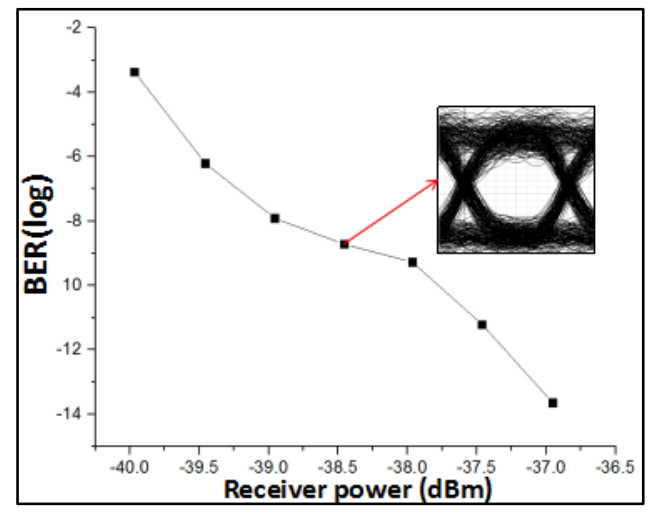

(a)

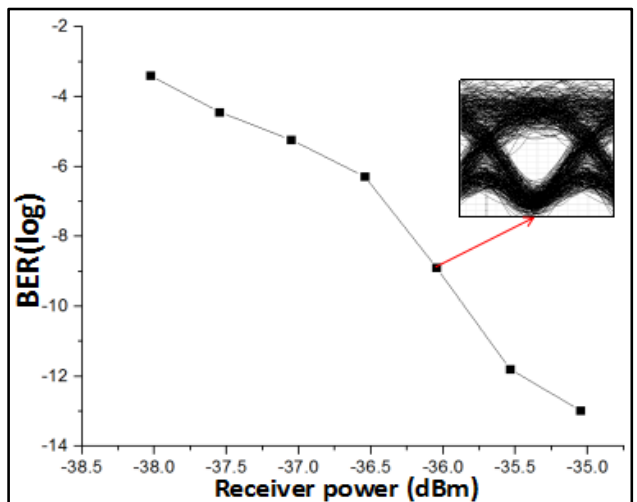

(b)

Fig. 4 The BER diagram of (a) LPF-ODB scheme and (b) DA-ODB scheme

\section{Summary}

As a novel wireless optical communication technology, VLC has a wide application value in modern communication systems. In this paper, a VLC system based on LED is designed. Two different optical optical duobinary modulation schemes are used, and the transmission distance is $10 \mathrm{~m}$. The simulation results verify the feasibility of optical duobinary modulation in the visible light communication system. By comparing the time domain waveforms, BER and eye diagrams, it is found that the DA-ODB scheme has better receiver sensitivity and better transmission performance. In addition, the results will provide reference for the future VLC system applications.

\section{Acknowledgements}

This work was financially supported by the National Natural Science Foundation of China (No.61107064), Innovation Program of Shanghai Municipal Education Commission (No.15ZZ101), Leading Academic Discipline Project of Information and Communication Engineering (No.XXKZD1605), School Foundation (No.EGD14XQD01) of Shanghai Polytechnic University, College Students' Science and Technology Innovation Project of Shanghai Polytechnic University (No.2016-xjkj-063), Connotation construction project of Shanghai Polytechnic University (No.A11NH170301).

\section{References}

[1] Song Xiaoqing, Zhao Zixu, Chen Kewei, Liu Zili. Application Prospect and Development Challenges of Visible Light Communication [J]. Advances in Laser and Optoelectronics, 2015, (08): 36-44.

[2] Peng Xiao. Free space visible light communication [J]. science, 2016, (11): 50-52.

[3] Huang X, Wang Z, Shi J, et al. $1.6 \mathrm{Gbit} / \mathrm{s}$ phosphorescent white LED based VLC transmission using a cascaded pre-equalization circuit and a differential outputs PIN receiver [J]. Optics Express, 2015, 23(17):22034-42.

[4] Trong-Hop Do, Junho Hwang, Myungsik Yoo, et al. Analys is of the effects of LED direction on the performance of visible light communication system [J]. Photonic network communications, 2013, 25(1):60-71. 
[5] Wireless World Research Forum. http://www.wireless-worldresearch.org.

[6] M. Saadi, L. Wattisuttikulkij, Y. Zhao, P. Sangwongngam. Visible Light Communication: Opportunities, Challenges and Channel Models[C]. International Journal of Electronics and Informatics, 2013,(02): 1-3.

[7] Lu Zhijia, Wang Xianbin, Li Li, et al. Analysis of Optical Binary Binary Transmission in High Speed Optical Fiber System [J]. Optical Communications Technology, 2015,(02): 34-37.

[8] FAN C X, ZHAN D Y, XU B X, et al. Communications Principles[M]. Beijing: Defense Industry Press, 1995.

[9] Shao Yufeng, Chen Lin, Wen Shuangchun, et al. Coding and decoding scheme of new improved optical double binary transmission [J]. Journal of Communications, 2007, 28(2): 58-63.

[10] Zhou Yue, Shao Yufeng, Wang Zhifeng, et al. Research on Dispersion Compensation of $40 \mathrm{~GB} / \mathrm{s}$ Optical Duo-Binary Coded Transmission System[J]. 2016, 06(8):190-195. 